\title{
DETERMINATENESS AND PARTITIONS
}

\author{
KAREL PRIKRY
}

\begin{abstract}
It is proved that the axiom of determinateness of Mycielski and Steinhaus for games in which players alternate in writing reals implies that $\omega \rightarrow(\omega)_{2}^{\omega}$ (i.e. for every partition of infinite sets of natural numbers into two classes there is an infinite set such that all its infinite subsets belong to the same class).
\end{abstract}

For every infinite $N \subseteq \omega, \Omega(N)$ denotes the family of infinite subsets of $N$. We also set $\Omega(\omega)=\Omega$.

A set $Q \subseteq \Omega$ is said to be Ramsey if there exists $N \in \Omega$ such that $\Omega(N) \subseteq \mathbb{Q}$ or $\Omega(N) \subseteq \Omega-\mathbb{Q}$.

It is easily shown that the existence of a non-Ramsey $\mathcal{Q} \subseteq \Omega$ follows from the axiom of choice. But Mathias [3] has proved that in Solovay's model [7] in which every set of reals is Lebesgue measurable and has the property of Baire, every set $\mathscr{Q} \subseteq \Omega$ is Ramsey. Silver [6] has proved that every analytic $\mathscr{Q} \subseteq \Omega$ is Ramsey. Ellentuck [1] has simplified Silver's proof and demonstrated that all sets in the least class containing all Borel sets and closed under the Suslin operation and complementation are Ramsey.

We shall show that a form of determinateness considered by Mycielski [4] implies that every $Q \subseteq \Omega$ is Ramsey. This will follow fairly easily from the results of Ellentuch [1] and Oxtoby [5].

We shall start by formulating the axiom $A_{R}^{* *}$, due to Mycielski [4], asserting the determinateness of a class of games introduced by him. Let $R$ denote the set of reals and $R^{\omega}$ the set of all $\omega$-sequences of reals. Let $Q \subseteq R^{\omega}$. The game $G_{R}^{* *}(\mathscr{Q})$ is played by players I and II who alternate in building an $\alpha \in R^{\omega}$ in the following way: I picks $\alpha_{0}, \ldots, \alpha_{n_{1}}\left(n_{1} \geqslant 0\right)$; II picks $\alpha_{n_{1}+1}, \ldots, \alpha_{n_{2}}$ $\left(n_{2}>n_{1}\right)$; I picks $\alpha_{n_{2}+1}, \ldots, \alpha_{n_{3}}$, etc. I wins if $\alpha \in \mathbb{Q}$ and II wins otherwise. $A_{R}^{* *}$ is the assertion that for every $\mathscr{Q} \subseteq R^{\omega}, G_{R}^{* *}(\mathscr{Q})$ is determined. This contradicts the axiom of choice (see [4]; this also follows from the Theorem below). Our main result is

THEOREM. ZF $+A_{R}^{* *} \vdash$ every $Q \subseteq \Omega$ is Ramsey.

We shall need some results of Ellentuck [1]. An exposition of these results follcws.

We consider $\Omega$ as a topological space with the topology defined in [1]. In order to describe this topology, we set for $A \in \Omega$ and $n \in \omega$

\footnotetext{
Received by the editors February 27, 1974 and, in revised form, May 29, 1975.

AMS (MOS) subject classifications (1970). Primary 04A20, 02K05.

${ }^{1}$ The preparation of this paper, during its various stages, was supported by NSF grant GP-43841, a Fellowship from the Institute for Advanced Study and a grant from the Science Research Council.
} 


$$
\Omega_{n}(A)=\{M \in \Omega: A \cap n=M \cap n \wedge M-n \subseteq A\} .
$$

These sets form a base for Ellentuck's topology on $\Omega$. From now on, "open" will mean open in this topology and, similarly, other topological terms will refer to this topology unless specified otherwise.

By definition, $Q \subseteq \Omega$ has the property of Baire if $Q=Q \triangle \mathcal{Q}$ where $\mathscr{Q}$ is open, $C$ is meager and $\triangle$ is the symmetric difference sign. $Q$ is said to be completely Ramsey if for every $A \in \Omega$ and every $\mathrm{n} \in \omega$ there exists a $B \in \Omega$ such that $B \subseteq A, B \cap n=A \cap n$ and either $\Omega_{n}(B) \subseteq Q$ or $\Omega_{n}(B) \subseteq \Omega-$ $Q$. It is clear that every completely Ramsey set has the property of Baire (no choice is needed). The main result of Ellentuck [1] is

LEMMA 1. If $Q \subseteq \Omega$ has the property of Baire, then $\Theta$ is completely Ramsey.

\section{Ellentuck has also shown}

LeMMA 2. Every meager set is nowhere dense.

The proofs of Lemmas 1 and 2 go through in $\mathrm{ZF}+\mathrm{DC}_{R}$, where $\mathrm{DC}_{R}$ is the axiom of dependent choices for relations on $R$.

By Lemma 1 and a preceding remark, completely Ramsey sets coincide with the sets having the property of Baire. It is a well-known fact that the property of Baire is preserved under the Suslin operation. Hence so is being completely Ramsey. Silver's and Ellentuck's theorems stated in the introduction now readily follow.

In general, some form of choice is needed to prove that the property of Baire is preserved under the Suslin operation. Due to the peculiar property of the space $\Omega$ expressed in Lemma $2, \mathrm{DC}_{R}$ suffices in the present context. We omit the details since this is not needed for our main result. By contrast, the axiom of choice for countable families of sets of reals suffices for the proof that the property of Baire for sets of reals, with respect to the usual topology, is preserved under the Suslin operation. This also holds true of Lebesgue measurability.

We shall also need a simple fact about Ellentuck's topology given by Lemma 4. The proofs of Lemmas 3 and 4 do not use the axiom of choice.

LEMMA 3. If $Q \subseteq \Omega$ does not have the property of Baire, then there is a

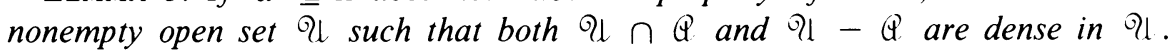

Proof. If $Q$ does not have the property of Baire we can find some nonempty open $\mathscr{Q}$ such that $Q-\operatorname{Int}(\mathcal{Q})$ is dense in $\mathscr{Q}$, where $\operatorname{Int}(Q)$ denotes the interior of $\mathscr{Q}$. Then $\mathscr{Q}$ is as desired.

LEMma 4. If some $Q \subseteq \Omega$ does not have the property of Baire, then for some $\Theta \subseteq \Omega$, both $\Theta$ and $\Omega-Q$ are dense in $\Omega$.

Proof. Let $Q \subseteq \Omega$ not have the property of Baire. Let $\mathcal{Q l}_{\mathrm{l}}$ be given by Lemma 3. We can clearly suppose that for some $A \in \Omega$ and $n \in \omega$, Q $=\Omega_{n}(A)$. Let $f$ be a one-to-one increasing map of $A-n$ onto $\omega$. Set $\mathbb{Q}^{\prime}=\left\{f^{*}(M-n): M \in \Omega_{n}(A)\right\}$. Then $\mathbb{Q}^{\prime}$ is as desired.

We shall also need a metamathematical result which is proved by using Cohen's method of forcing. Let $\forall_{1}^{2}$ be the set of all sentences equivalent in ZF 
to a sentence of the form $(\forall X \subseteq R) \Phi$, where $\Phi$ involves only quantifiers over real numbers.

LEMMA 5. If $\sigma \in \forall_{1}^{2}$ and $\mathrm{ZF}+(R$ can be well ordered $) \vdash \sigma$, then $\mathrm{ZF}+$ $\mathrm{DC}_{R} \vdash \sigma$.

Proof. The following fact is well known and not difficult, and readily implies the lemma: Every countable transitive model $M$ of $\mathrm{ZF}+\mathrm{DC}_{R}$ has a generic extension to a model $N$ of $\mathrm{ZF}+(R$ can be well ordered $)$ such that $R^{(N)}=R^{(M)}$.

To obtain $N$, we consider in $M$ the tree $T$ of well-orderings of countable subsets of $R . w_{1}$ precedes $w_{2}$ in $T$ iff $w_{1}$ is an initial segment of $w_{2} . N$ is obtained from $M$ by adding a generic branch of $T$. Since in $M$ there is a coding $R \rightarrow T$, we can use $\mathrm{DC}_{R}$ to check that $R^{(N)}=R^{(M)}$.

REMARK. In Lemma 5, " $R$ can be well ordered" could be replaced by $\mathrm{CH}$; indeed, the above proof yields an extension $N$ which satisfies $\mathrm{CH}$.

The main application of Lemma 5 follows.

LEMMA $\epsilon$.

$$
\mathrm{ZF} A_{R}^{* *} \vdash \forall Q \subseteq \Omega \exists A \in \Omega \exists n \in \omega\left[\Omega_{n}(A) \subseteq Q \vee \Omega_{n}(A) \subseteq \Omega-Q\right]
$$

Proof. Let $Q \subseteq \Omega$. Let $\Gamma(\Theta)$ denote the game in which players I and II alternately pick basic open sets $\Omega_{k_{n}}\left(A_{n}\right)$ in such a way that $\Omega_{k_{n+1}}\left(A_{n+1}\right)$ $\subseteq \Omega_{k_{n}}\left(A_{n}\right),(n \in \omega)$. $\Omega_{k_{n}}\left(A_{n}\right)$ is picked by I if $n$ is even by II if $n$ is odd. I wins if $\left(\cap\left\{\Omega_{k_{n}}\left(A_{n}\right): n \in \omega\right\}\right) \cap \mathbb{Q} \neq 0$. II wins otherwise.

Let $\sigma_{0}$ be the sentence

$$
\forall \mathscr{Q} \subseteq \Omega\left[\operatorname{Det}(\Gamma(\mathscr{Q})) \rightarrow \exists A \in \Omega \exists n \in \omega\left[\Omega_{n}(A) \subseteq \mathbb{Q} \vee \Omega_{n}(A) \subseteq \Omega-\mathbb{Q}\right]\right],
$$

where $\operatorname{Det}(\Gamma(\mathbb{Q}))$ stands for “ $\Gamma(\mathbb{Q})$ is determined". Since $\operatorname{Det}(\Gamma(\mathbb{Q}))$ has only an existential quantifier over strategies it follows that $\sigma_{0} \in \forall_{1}^{2}$. We claim that

$$
\mathrm{ZF}+(R \text { can be well ordered }) \vdash \sigma_{0} \text {. }
$$

From the results of Oxtoby [5] we conclude: If II has a winning strategy in $\Gamma(\mathbb{Q})$, then $Q$ is meager. If I has a winning strategy in $\Gamma(Q)$, then

$$
\exists \mathscr{U} \subseteq \Omega[\mathscr{Q} \neq 0 \wedge \mathscr{Q} \text { is open } \wedge(\mathscr{Q}-\mathscr{Q}) \text { is meager }] \text {. }
$$

Oxtoby [5] uses the axiom of choice, but it is easy to see that for his results to go through in the present context, all one needs is that a well-ordering of $R$ exists. The claim now follows immediately from Lemma 2.

Hence by Lemma $5, \mathrm{ZF}+\mathrm{DC}_{R} \vdash \sigma_{0}$. Finally, it is not hard to see that $\Gamma(\mathbb{Q})$ is equivalent to $G_{R}^{* *}\left(\mathbb{Q}^{\prime}\right)$ for a suitable $Q^{\prime}$. Hence $A_{R}^{* *}$ implies $\operatorname{Det}(\Gamma(\mathscr{Q}))$ and Lemma 6 follows.

Proof of the Theorem. By Lemmas 4 and 6 , every $Q \subseteq \Omega$ has the property of Baire. Now we use Lemma 1.

REMARKS. It is not known whether the Theorem remains true if $A_{R}^{* *}$ is replaced by the standard axiom of determinateness $A_{\omega}$.

In the first version of this paper the Theorem was proved under the assumption $A_{R}+$ DC. J. Silver pointed out that DC is not needed and J. Mycielski pointed out that $A_{R}$ can be replaced by $A_{R}^{* *}$. J. Mycielski and W. Reinhardt also suggested numerous other improvements. In particular, they 
recommended that the underlying principle behind the proof of Lemma 6 should be isolated explicitly in Lemma 5.

\section{REFERENCES}

1. E. Ellentuck, A new proof that every analytic set is Ramsey, J. Symbolic Logic 39 (1974), $163-165$.

2. F. Galvin and K. Prikry, Borel sets and Ramsey's theorem, J. Symbolic Logic 38 (1973), 193-198. MR 49 \#2399.

3. A. R. D. Mathias, On a generalization of Ramsey's theorem, Thesis, Bonn, 1968.

4. J. Mycielski, On the axiom of determinateness. II, Fund. Math. 59 (1966), 203-212. MR 35 \# 1489 .

5. J. C. Oxtoby, The Banach-Mazur game and Banach category theorem, Contributions to the Theory of Games, vol. 3, Ann. of Math. Studies, no. 39, Princeton Univ. Press, Princeton, N.J., 1957, pp. 159-163. MR 20 \#264.

6. J. H. Silver, Every analytic set is Ramsey, J. Symbolic Logic 35 (1970), 60-64. MR 48 \#10807.

7. R. M. Solovay, A model of set-theory in which every set of reals is Lebesgue measurable, Ann. of Math. (2) 92 (1970), 1-56. MR 42 \#64.

Ddpartment of Mathematics, University of Cambridge, Cambridge, England

Current address: Department of Mathematics, University of Minnesota, Minneapolis, Minnesota 55455 